\title{
Linezolid, vancomycin and daptomycin modulate cytokine production, Toll-like receptors and phagocytosis in a human in vitro model of sepsis
}

\author{
Christian Bode ${ }^{1,4}$, Stefan Muenster ${ }^{1,4}$, Britta Diedrich ${ }^{1,2}$, Sebastian Jahnert ${ }^{1}$, Christina Weisheit ${ }^{1}$, \\ Folkert Steinhagen ${ }^{1}$, Olaf Boehm ${ }^{1}$, Andreas Hoeft ${ }^{1}$, Rainer Meyer ${ }^{3}$ and Georg Baumgarten ${ }^{1}$
}

Conventional antibiotics exhibit immunomodulatory properties beneficial in the treatment of sepsis. Antibiotic-resistant Grampositive bacteria have become a problem in sepsis therapy, giving rise to increased use of last-resort antibiotics; for example, linezolid (LIN), vancomycin (VAN) and daptomycin (DAP). As the immunomodulatory properties of these antibiotics in treating sepsis are unknown, this study examined the effect of VAN, LIN and DAP on the immune response under sepsis-like conditions in vitro. Lipopolysaccharide (LPS)-activated THP-1 monocytes were incubated with LIN, VAN or DAP. Gene expression of

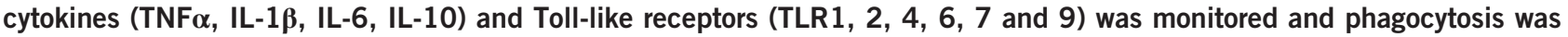
determined following coincubation with $E$. coli. The antibiotics differentially modulated the gene expression of the investigated cytokines. While LIN and VAN upregulated the expression of all TLRs, DAP downregulated mRNA levels of TLR1, TLR2 and TLR6, which recognize pathogen-associated molecular patterns from Gram-positive bacteria. In addition, LIN inhibited, whereas VAN promoted the phagocytic activity of monocytes. Our results suggest that LIN and VAN possess pro-inflammatory properties, whereas DAP might reduce the immune response to Gram-positive bacteria in sepsis. Furthermore, VAN might be beneficial in the prevention of Gram-negative infections by increasing the phagocytosis of $E$. coli.

The Journal of Antibiotics (2015) 68, 485-490; doi:10.1038/ja.2015.18; published online 4 March 2015

\section{INTRODUCTION}

Sepsis is the most common cause of death among critically ill patients in non-cardiac intensive care units. ${ }^{1}$ It is characterized by a systemic inflammatory response resulting from excessive stimulation of innate immune cells with molecular structures from microbial pathogens. These 'pathogen-associated molecular patterns' (PAMPs), ${ }^{2}$ such as lipopolysaccharide (LPS), are recognized by pattern recognition receptors, including Toll-like receptors (TLRs), RIG-like receptors, C-type lectin receptors and Nod-like receptors. ${ }^{3}$ Among pattern recognition receptors, TLRs have an outstanding role in primary recognition of microorganisms. They activate signaling cascades that lead to the induction of pro-inflammatory cytokines, such as TNF $\alpha$, IL-1 $\beta$ and IL-6, and further trigger the clearance of pathogens by phagocytosis. ${ }^{4-6}$ Due to their crucial role in sepsis, research has particularly focused on the modulation of TLRs. Increasing evidence now suggests that TLRs could be a potential target for the treatment of sepsis. ${ }^{7,8}$

Within the last two decades major advances have been made in understanding the pathophysiology of sepsis. However, clinical trails have failed to establish new therapeutic concepts and hence, classical anti-infective strategies with antibiotics remain one of the few effective treatments for sepsis. In addition to their antimicrobial activity, there is growing evidence that certain antibiotics exert their beneficial effects in inflammatory diseases directly by modulating the immune system. ${ }^{9,10}$ In this context, the first studies have already shown that broad-spectrum antibiotics modulate the immune response under septic conditions, thereby improving the outcome of individuals in both experimental and clinical settings. ${ }^{11-14}$

An increase in bacterial resistance over time and especially antibiotic-resistant Gram-positive bacteria, such as methicillinresistant Staphylococcus aureus, has unfortunately arisen due to frequent and inadequate use of broad-spectrum antibiotics. This has meanwhile become a major problem in the treatment of sepsis. ${ }^{1}$ Often, multidrug-resistant bacterial infections can only be treated with so-called 'last-resort antibiotics'. Currently, the glycopeptide antibiotic vancomycin (VAN) and recently developed antibacterials, daptomycin (DAP) and linezolid (LIN), represent leading options in the therapy of infections caused by multidrug-resistant Gram-positive bacteria. ${ }^{15}$

However, this emerging development in the anti-infective therapy of sepsis makes necessary greater exploration of the interplay between last-resort antibiotics and the immune system. As the immunomodulatory effects of these types of antibiotics in sepsis have so far been only

${ }^{1}$ Department of Anesthesiology and Intensive Care Medicine, University Hospital Bonn, Bonn, Germany; ${ }^{2}$ Department of Dermatology, University Medical Center Freiburg, Freiburg, Germany and ${ }^{3}$ Institute of Physiology II, University of Bonn, Bonn, Germany

${ }^{4}$ These authors contributed equally to this work.

Correspondence: Dr C Bode, Department of Anesthesiology and Intensive Care Medicine, University Hospital Bonn, Sigmund-Freud-Straße 25, 53127 Bonn, Germany. E-mail: christian.bode@ukb.uni-bonn.de

Received 7 September 2014; revised 14 December 2014; accepted 19 December 2014; published online 4 March 2015 
minimally investigated, this study explored the effect of VAN, LIN and DAP on the innate immune response, including TLR expression and phagocytic activity, in a well-established in vitro model of human sepsis. We found that both LIN and VAN broadly upregulate the expression of TLRs. In contrast, DAP downregulates TLR1, TLR2 and TLR6, which recognize PAMPs from Gram-positive bacteria. Results further demonstrate that LIN inhibits whereas VAN promotes the phagocytic activity of monocytes in sepsis-like conditions.

\section{MATERIALS AND METHODS}

\section{Reagents and THP-1 cells}

Antibiotics were purchased from the following manufacturer: LIN from Pfizer (Berlin, Germany); VAN from Fresenius Kabi (Bad Homburg, Germany), DAP from Novartis (Nuremberg, Germany). LPS from E. coli (serotype 055:B5) was obtained from Sigma-Aldrich (Taufkirchen, Germany). Human monocytic THP-1 cells (ATCC TIB-202) were maintained in RPMI-1640 medium (PAA, Coelbe, Germany) supplemented with 10\% FCS (Biochrom, Berlin, Germany), $0.05 \mathrm{~mm}$ 2-mercaptoethanol (Sigma-Aldrich), $100 \mu \mathrm{g} \mathrm{ml}^{-1}$ penicillin, and $100 \mathrm{mg} \mathrm{ml}^{-1}$ streptomycin (both from Invitrogen, Frankfurt, Germany). The cells were cultured in a suspension free of both antibiotics and FCS for $48 \mathrm{~h}$ before experimental use to avoid any confounding stimulation that might result from antibiotics or endotoxins contained in the cell culture medium. The viability of monocytes was ascertained using the tryptan blue staining method. Cell cultures were selected for the following experiments if viability was over $90 \%$.

\section{Stimulation of THP-1 cells}

Cells were stimulated at a concentration of $1 \times 10^{6}$ cells per $\mathrm{ml}$ in 6-well plates (Cellstar, greiner bio-one, Frickenhausen, Germany) in the presence of LPS $\left(10 \mu \mathrm{g} \mathrm{ml}^{-1}\right)$, LIN $\left(20 \mu \mathrm{g} \mathrm{ml}^{-1}\right)$, VAN $\left(50 \mu \mathrm{g} \mathrm{ml}^{-1}\right)$, DAP $\left(3 \mu \mathrm{g} \mathrm{ml}^{-1}\right)$, or via a combination of LPS with each individual antibiotic. The THP-1 cells were incubated at $37{ }^{\circ} \mathrm{C}$ and were removed from the culture plates after 2, 6 and $24 \mathrm{~h}$ to isolate mRNA.

\section{Isolation of mRNA and real-time PCR}

Total mRNA was isolated from the THP-1 cells using the RNeasy mini kit (Qiagen, Hilden, Germany) according to the manufacturer's instructions. cDNA was synthesized with the High Capacity cDNA Reverse Transcription Kit according to the manufacturer's instructions (Applied Biosystems, Darmstadt, Germany). Real-time PCR was carried out on the 7900HT Fast Real-Time PCR system using TaqMan Gene Expression Assays primer/probe sets (all from Applied Biosystems) and the standard thermal-cycling conditions for relative quantification designed by the manufacturer. Quantification of the PCR signals for each sample was done by comparing the cycle threshold values in duplicate for the gene of interest with the cycle threshold values for the GAPDH housekeeping gene. The mean relative mRNA expression was determined by using SDS software V2.2 (Applied Biosystems).

\section{Phagocytosis assay}

Phagocytosis by THP-1 cells was monitored using the pHrodo E. coli BioParticles Conjugate assay (Invitrogen) according to the manufacturer's instructions. In short, to prepare BioParticles, $2 \mathrm{ml}$ of phosphate-buffered saline was used for reconstitution. THP- 1 cells $\left(1 \times 10^{6}\right.$ cells per ml $)$ were incubated in LIN, VAN or DAP in the presence of LPS for $24 \mathrm{~h}$ as described above, followed by an incubation with pHrodo E. coli BioParticles for $2 \mathrm{~h}$. DNA staining for viability assessment was performed before FACS with Hoechst $33342\left(2 \mu \mathrm{g} \mathrm{ml}^{-1}\right.$, Invitrogen). Flow cytometry was performed using a Canto III Flow Cytometer System (BD Biosciences, Heidelberg, Germany) and FlowJo Analysis Software (Treestar, Ashland, OR, USA). The phagocytosis of E. coli by LPS-activated monocytes was set as $100 \%$ and the percent change observed in cells treated with LPS plus antibiotics calculated.

\section{Statistical analysis}

Statistical analysis was conducted using GraphPad Prism 5 (GraphPad Software, La Jolla, CA, USA). Mann-Whitney $U$ test was used to examine differences in phagocytic assay and in gene expression of cytokines and TLRs. Values of $P<0.05$ were considered to be statistically significant. All data are expressed as mean \pm s.e. unless otherwise noted.

\section{RESULTS}

LIN, VAN and DAP differentially regulate the gene expression of sepsis-associated cytokines

Cytokines such as TNF $\alpha$, IL-1 $\beta$, IL- 6 and IL-10 have a crucial role in the inflammatory response during sepsis. ${ }^{16}$ To determine a potential effect of LIN, VAN and DAP on the expression of sepsis-associated cytokines, we incubated human THP-1 monocytes with clinically relevant concentrations of LIN, VAN or DAP in the presence or absence of LPS. ${ }^{17-19}$ The expression of genes encoding for TNF $\alpha$, IL-1 $\beta$, IL- 6 and IL-10 was analyzed by RT-PCR after 2, 6 and $24 \mathrm{~h}$. We found that all three tested antibiotics showed differential regulatory effects on gene expression of the examined cytokines. Specifically, LIN greatly increased the expression of proinflammatory cytokines TNF $\alpha$, IL-1 $\beta$ and IL- 6 compared with unstimulated controls at 6 and $24 \mathrm{~h}$ (Figures 1a-c and Supplementary Figures S1B, D and F) whereas the mRNA level of the anti-inflammatory cytokine IL-10 was significantly reduced at $24 \mathrm{~h}$ (Figure 1d). In co-stimulation with LPS, LIN significantly upregulated the gene expression of TNF $\alpha$ compared with the LPS-stimulated control at 6 and $24 \mathrm{~h}$ (Figure 1a and Supplementary Figure S1B). Furthermore, mRNA levels of IL-6 were increased by LIN at all investigated time points and IL- $1 \beta$ gene expression was significantly upregulated at 2 and $6 \mathrm{~h}$ (Figure 1c and Supplementary Figures S1C-F). Interestingly (and in contrast to the LPS condition), IL-10 gene expression was upregulated by LIN in the presence of LPS at all investigated time points (Figure $1 \mathrm{~d}$ and Supplementary Figure S1G and $\mathrm{H}$ ). The incubation of monocytes with VAN increased the mRNA level of TNF $\alpha$ and IL-6 compared with unstimulated controls at 6 and $24 \mathrm{~h}$ (Figures $1 \mathrm{a}$ and $\mathrm{c}$ and Supplementary Figures S1B and D). The co-stimulation with VAN and LPS upregulated the gene expression of TNF $\alpha$ compared with LPS controls at 6 and $24 \mathrm{~h}$ (Figure 1a and Supplementary Figure S1B), IL-6 and IL-10 mRNA levels were significantly elevated in LPS-activated monocytes by VAN at all time points (Figures $1 \mathrm{c}$ and $\mathrm{d}$ and Supplementary Figures S1C-H). The incubation of unstimulated THP-1 cells with DAP upregulated gene expression of IL-6 and downregulated the IL-10 mRNA levels at $24 \mathrm{~h}$ (Figures $1 \mathrm{c}$ and d). Monocytes stimulated with DAP \pm LPS exhibited significantly higher mRNA level of IL-6 (24 h, Figure 1c) and IL-10 (2 h, Supplementary Figure S1G), whereas the mRNA level of IL- $1 \beta$ was considerably reduced compared with LPS-activated cells at $24 \mathrm{~h}$ (Figure 1b).

\section{LIN, VAN and DAP differentially regulate the gene expression of TLRs}

Since signaling via TLRs is a first-line of innate immune defense against microbial pathogens in sepsis, ${ }^{2}$ we examined immunomodulatory effects of LIN, VAN and DAP on expression of TLRs. Human THP-1 cells were stimulated with LIN, VAN or DAP with and without LPS. TLR expression was monitored by RT-PCR after 2, 6 and $24 \mathrm{~h}$.

As seen in Figure 2 and Supplementary Figure S2, the monostimulation of cells with LIN significantly increased the gene expression of TLR1 (at 2, 6 and 24h), TLR2 (at 6 and $24 \mathrm{~h}$ ), TLR4 (at $6 \mathrm{~h}$ ), TLR6 (at 2 and $24 \mathrm{~h}$ ), TLR7 (6h) and TLR9 (at $24 \mathrm{~h}$ ) compared with unstimulated controls at the indicated time points. When LIN was administered to LPS-activated cells, the highest changes in TLR gene expression were observed after $24 \mathrm{~h}$ of incubation (Figure 2). At this time point, all investigated TLRs were upregulated compared with LPS-activated controls (Figures 2a-f). The incubation of THP-1 cells 
LPS

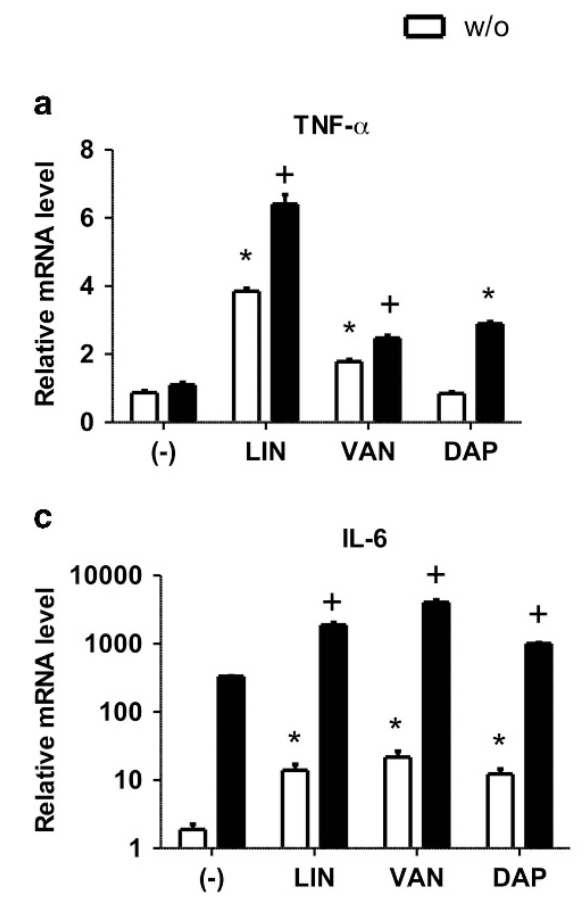

C

$(-)$

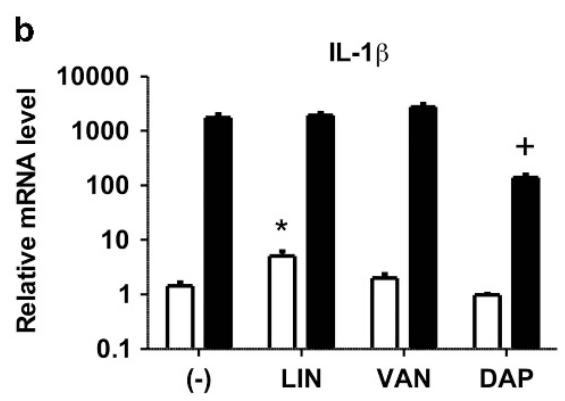

d

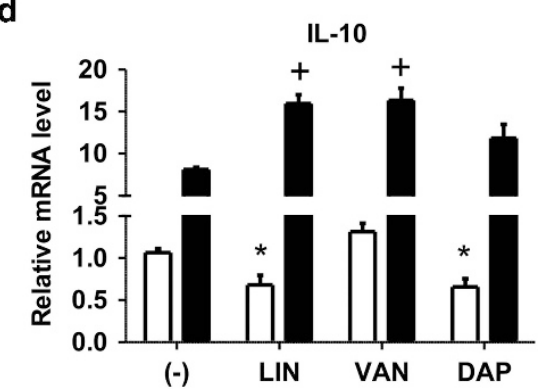

Figure 1 Linezolid (LIN), vancomycin (VAN) and daptomycin (DAP) differentially modulate the expression of cytokines in monocytes. THP-1 monocytes were incubated with LIN, VAN or daptomycin in the presence or absence of lipopolysaccharide (LPS) for $24 \mathrm{~h}$. Quantitative real-time-PCR (qRT-PCR) analysis was performed to determine the expression levels of genes encoding TNF $\alpha$ (a), IL-1 $\beta$ (b), IL-6 (c) and IL-10 (d) after normalization to GAPDH. Each bar represents the fold change in mRNA expression comparing unstimulated control with antibiotic-incubated cells or LPS-stimulated control with LPS plus antibiotic-stimulated cells, respectively. Data are expressed as mean \pm s.e.m. of six independent experiments; ${ }^{*} P<0.05$ vs respective LPS-free control; $+P<0.05$ vs respective LPS control.

with VAN increased the expression of all investigated TLRs, except for TLR9, compared with unstimulated controls at various time points (TLR1: 2, 6 and 24 h; TLR2: 6 and 24 h; TLR4: 2, 6 and 24h; TLR6: 2 and 24h; TLR7: 6 and 24 h; Figure 2 and Supplementary Figure S2). In LPS-activated monocytes, VAN induced upregulation of both TLR1, TLR4, TLR7 at $6 \mathrm{~h}$ and TLR1, TLR2, TLR4, TLR7 compared with their respective controls at $24 \mathrm{~h}$ (Figures $2 \mathrm{a}-\mathrm{c}$ and $\mathrm{e}$ and Supplementary Figures S2B, F and J).

In contrast to LIN and VAN, the incubation of THP-1 cells with DAP reduced gene expression of TLR1 compared with unstimulated monocytes at $24 \mathrm{~h}$ (Figure $2 \mathrm{a}$ ).

In LPS-activated cells, DAP differentially regulated the gene expression of TLRs: DAP downregulated the gene expression of TLR1, TLR2, TLR6 and TLR9 while upregulating TLR4 and TLR7 gene expression (Figure 2 and Supplementary Figure S2).

\section{LIN and VAN modulate the bacterial phagocytosis}

Previous work has established that TLRs have a considerable role in regulating the phagocytosis of bacteria. ${ }^{6}$ After observing an effect of LIN, VAN and DAP on the expression of TLRs, we examined the effect of these antibiotics on the phagocytotic activity. Therefore, THP-1 monocytes were stimulated with LIN, VAN or DAP in the presence of LPS as described above, followed by incubation with heatkilled E. coli. As shown in Figure 3, LIN in combination with LPS significantly reduced the phagocytotic activity by $25 \%$ compared with LPS-activated controls. When monocytes were co-incubated with LPS and VAN, the phagocytosis of E. coli was significantly increased by more than $20 \%$ compared with the monostimulation with LPS, while DAP had no effect on the phagocytosis of E. coli by monocytes.

\section{DISCUSSION}

Last-resort antibiotics LIN, VAN and DAP, commonly used to treat multidrug-resistant Gram-positive sepsis, differentially modulate the innate immune response in the absence and presence of endotoxin. We found that both LIN and VAN broadly upregulate the expression of distinct TLRs. In contrast, DAP downregulates TLR1, TLR2 and TLR6, which are responsible for the recognition of PAMPs from Gram-positive bacteria. Furthermore, the phagocytic activity of monocytes in sepsis-like conditions is inhibited by LIN inhibits, yet is promoted by VAN.

TLRs have a critical role in the pathophysiology of sepsis by detecting PAMPs from bacteria and subsequently stimulating cells of the innate immune system such as monocytes to produce proinflammatory molecules including TNF $\alpha$, IL1 $\beta$ and IL-6., ${ }^{4,5}$ TLR2 interacts functionally with both TLR1 and TLR6 and binds PAMPs from Gram-positive bacteria. ${ }^{4}$ For the first time, we demonstrate that VAN and LIN upregulate the expression of TLR1, TLR2 and TLR6 whereas DAP downregulates these TLRs under sepsis-like conditions. TLR9, which elicits an immune response after binding to bacterial CpG-DNA, was also downregulated by DAP in LPS-activated monocytes in contrast to LIN and VAN incubated cells. Hence, we conclude that therapy with VAN or LIN may elicit a stronger immune response after binding PAMPs from Gram-positive bacteria compared with DAP due to the upregulation of the aforementioned TLRs. Consistent with this, English et al. ${ }^{20}$ found that DAP reduces the inflammatory response of macrophages to $S$. aureus in vitro compared with VAN. This implies downregulation of TLR1, TLR2, TLR6 and TLR9 expression by DAP (and the subsequent reduced recognition of PAMPs released by $S$. aureus) as the underlying mechanism for this observation. ${ }^{20}$ 


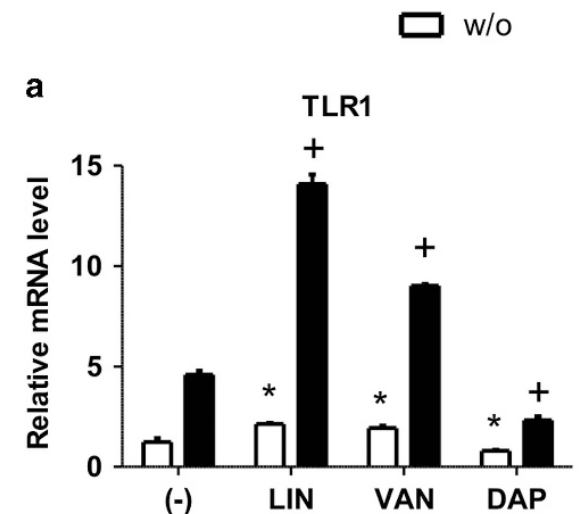

b
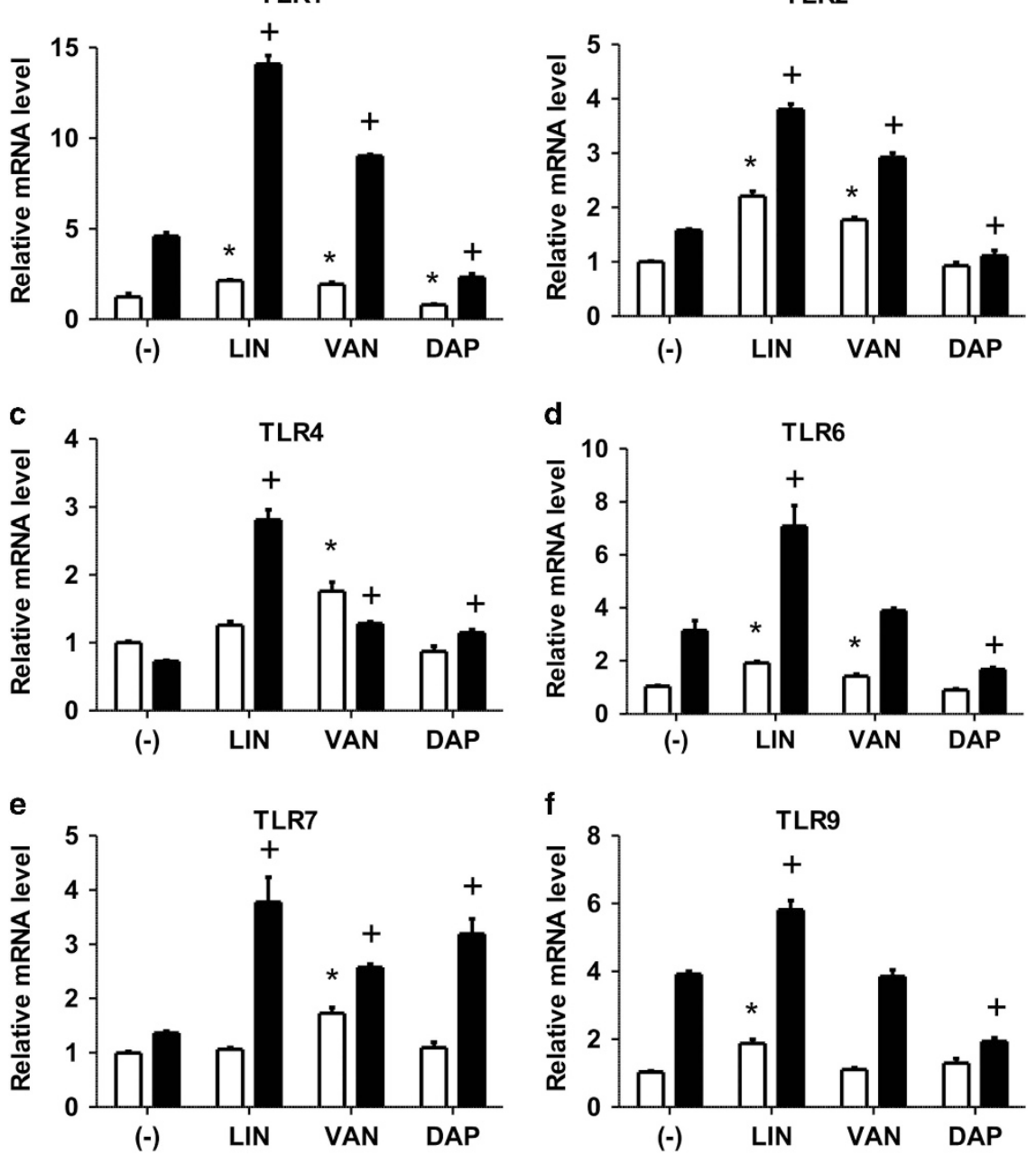

Figure 2 Linezolid (LIN), vancomycin (VAN) and daptomycin (DAP) differentially modulate the expression of Toll-like receptors (TLRs) in monocytes. THP-1 monocytes were incubated with LIN, VAN or DAP in the presence or absence of lipopolysaccharide (LPS) for $24 \mathrm{~h}$. Quantitative real-time-PCR (qRT-PCR) analysis was performed to determine the expression levels of genes encoding TLR1 (a), TLR2 (b), TLR4 (c), TLR6 (d), TLR7 (e) and TLR9 (f) after normalization to GAPDH. Each bar represents the fold change in mRNA expression comparing unstimulated control with antibiotic-incubated cells or LPSstimulated control with LPS plus antibiotic-stimulated cells, respectively. Data are expressed as mean \pm s.e.m. of six independent experiments; ${ }^{*} P<0.05$ vs respective LPS-free control; ${ }^{+} P<0.05$ vs respective LPS control.

Current results indicate that phagocytic activity of LPS-activated monocytes can be inhibited by LIN yet to be promoted by VAN regardless of their effects on TLR expression. This finding suggests that both LIN and VAN might regulate the internalization of bacteria by modulating further phagocytic receptors such as scavenger receptors or C-type lectin receptors. TLR-independent regulation of phagocytotic activity has been demonstrated for the immunomodulatory agent resveratrol. ${ }^{21}$ Resveratrol inhibited phagocytic activity of THP-1 monocytes by reducing scavenger receptor and CTRs expression independent of TLR signaling. Consistent with our current findings, the inhibition of the phagocytic activity by LIN has recently been shown for the internalization of $E$. coli in human neutrophils. ${ }^{22}$ Our results suggest that the therapy with LIN could lead to a higher incidence of Gram-negative infections compared to treatment with VAN or DAP. Of interest, Wilcox et al. ${ }^{23}$ observed a higher mortality in LIN-treated patients with catheter-related bloodstream infections, who were infected with Gram-negative organisms while patients with
Gram-positive infections showed no difference in mortality between VAN- and LIN-treated individuals. This led the FDA to issue an alert advising clinicians that LIN is not approved for treating (catheter-related bloodstream) infections caused by Gram-negative bacteria (US Food and Drug Administration. Information for Healthcare Professionals: Linezolid (marketed as zyvox) 3/16/2007. Available at: http://www.fda.gov/Drugs/DrugSafety/PostmarketDrugSafetyInformationforPatie ntsandProviders/DrugSafetyInformationforHeathcareProfessionals/ucm085249.htm, accessed 8 April 2014). Yet, more studies would be necessary to establish an association between current findings on bacterial phagocytosis and adverse effects of LIN in Gram-negative infections.

Although our results do not confirm the effects of VAN, LIN and DAP on the immune response at protein levels, several reports have found associations between change in protein expression and the regulation of the investigated mRNA for TLRs and cytokines. ${ }^{24-26}$ Thus, we conclude that VAN, LIN and DAP modulate the immune 


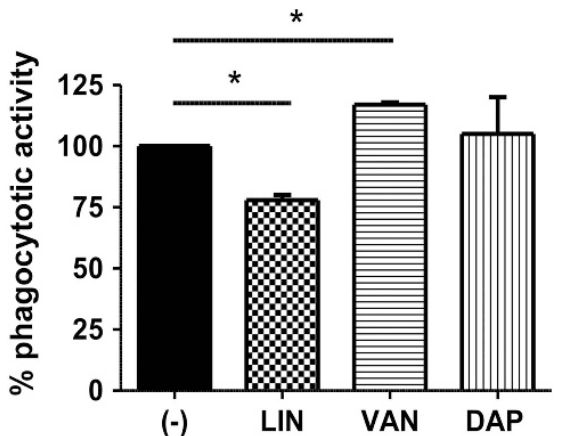

Figure 3 Effect of linezolid (LIN), vancomycin (VAN) and daptomycin (DAP) on the phagocytotic activity monocytes. THP-1 cells were incubated with LIN, VAN or DAP in the presence of lipopolysaccharide (LPS) as described in Figure 1. Thereafter, monocytes were incubated for $2 \mathrm{~h}$ with heat-killed rhodamine-conjugated $E$. coli and phagocytosis was determined by flow cytometry. The phagocytosis of E. coli by LPS-activated monocytes was defined as $100 \%$ (baseline) and the percent change observed in cells treated with LPS+antibiotics was calculated. Data are expressed as mean \pm s.d. of three independent experiments; ${ }^{*} P<0.05$.

system differentially and reliably. Consistent with this, our group recently investigated the immunomodulatory effects of commonly used classes of antibiotics under septic conditions. There, we identified chinolones (moxifloxacin), tetracyclines (doxycycline) and macrolides (erythromycin) as immunomodulators that regulate the expression of TLRs and cytokines. ${ }^{11}$ Current study extended the knowledge about the immunomodulatory potential of antibiotics and encourages the use of last-line antibiotics as immunomodulators in sepsis caused by Gram-positive organisms.

In line with our findings, previous reports demonstrated that VAN upregulates the gene expression of IL-10 and LIN induces greater production of IL-1 $\beta$, respectively. ${ }^{27,28}$ In contrast, VAN decreases the production of TNF $\alpha$, LIN reduces the production of proinflammatory cytokines, including IL- $1 \beta$, while DAP has no immunomodulatory effect on proinflammatory cytokines in the presence of LPS. ${ }^{27,29,30}$ These studies investigated the effects of antibiotics on whole blood samples such as peripheral blood mononuclear cells (PBMCs), while the current study focused on one key immune cell type. Distinct antibiotic immunomodulatory effects on monocytes and heterogeneous leukocyte populations (which include lymphocytes, monocytes, and dendritic cells) have been observed before. For example, it was shown that the antibiotic ciprofloxacin reduces the pro-inflammatory cytokine response to LPS in monocytes, whereas the treatment of a mixed cell population with ciprofloxacin increases their expression. ${ }^{31,32}$ These results indicate that cellular interactions could modify monocytic TLR and cytokine expression to a modulation by antibiotics.

Several antibiotics including the cell wall biosynthesis inhibitor VAN and the membrane damaging agent DAP may elicit a massive release of PAMPs by disrupting the bacterial cell, while bacteriostatic antibiotics such as the bacterial protein synthesis inhibitor LIN leads to the release of smaller quantities of PAMPs. The recogniton of PAMPs that are released by antibiotic treatment induces a strong inflammatory response, also known as the Jarisch-Herxheimer reaction. In addition to this indirect immunomodulatory effect of antibiotcs via PAMPs, the currrent study investigated the direct effect of LIN, VAN and DAP on the immune reponse toward LPS. In contrast to the potential indirect pro-inflammatory effects, LIN demonstrated a higher direct proinflammatory potential compared with DAP. We and other groups have demonstrated that other bacterial protein synthesis inhibitors such as gentamicin have no pro-inflammatory properties, ${ }^{11,28}$ suggesting that the effect of LIN on bacterial protein synthesis appears to not be critical for its immunomodualtory effects.

The molecular mechanisms that underlie these direct immunomodulatory effects of the investigated antibiotics are not well understood. This study shows that LIN increases the expression of all investigated cytokines and TLRs. A recent study reported that LIN activates the Nlrp3 inflammasome, thereby inducing a pro-inflammatory response both in vitro and in vivo. ${ }^{28}$ To elicit this immune response, LIN induces a mitochondrial dysfunction, which activates the Nlrp3 inflammasome via the mitochondrial lipid cardiolipin. This finding suggests that no direct interaction between LIN and the pattern recognition receptor Nlrp3 exists. Consistent with this, we found that LIN does not directly activate any TLRs by monitoring the immune response of TLR-transfected HEK cells after incubation with LIN (data not shown). Compared with LIN, DAP displays weaker regulatory effects on the innate immune response. This observation might be due to the very hydrophilic, water-soluble core of DAP. This characteristic might reduce the ability of DAP to penetrate human cells in significant amounts, and, subsequently, interaction with pattern recognition receptors or other molecules of the immune system. ${ }^{33}$ Thus, DAP might be beneficial in the antibiotic therapy of septic patients with a pre-existing strong (and harmful) pro-inflammatory response compared with VAN and LIN.

This study provides the first experimental evidence that last-resort antibiotics LIN, VAN and DAP modulate cytokine production, TLRs and phagocytosis in a human in vitro model of sepsis. It further shows that LIN and, especially, VAN have important pro-inflammatory effects on the innate immune system in contrast to DAP under sepsislike conditions. Of note, we found that LIN and VAN differentially modulate the phagocytosis of bacteria, suggesting differences between the treatment of VAN and LIN in the susceptibility for Gram-negative infections. Our present results together with future explorations of the immunomodulatory effects of LIN, VAN and DAP may serve as the basis for a new therapeutic use of the investigated antibiotics as immunomodulators in sepsis caused by Gram-positive organisms.

\section{CONFLICT OF INTEREST}

The authors have received financial support from Novartis. The funder did not have any involvement in protocol design, collection of data, statistical analysis and interpretation of findings.

1 Mayr, F. B., Yende, S. \& Angus, D. C. Epidemiology of severe sepsis. Virulence 5, 4-11 (2014).

2 Rittirsch, D., Flierl, M. A. \& Ward, P. A. Harmful molecular mechanisms in sepsis. Nat. Rev. Immunol. 8, 776-787 (2008).

3 Kumar, H., Kawai, T. \& Akira, S. Pathogen recognition by the innate immune system. Int. Rev. Immunol. 30, 16-34 (2011).

4 Kawai, T. \& Akira, S. The role of pattern-recognition receptors in innate immunity: update on Toll-like receptors. Nat. Immunol. 11, 373-384 (2010).

5 van der Poll, T. \& Opal, S. M. Host-pathogen interactions in sepsis. Lancet 8, 32-43 (2008).

6 Blander, J. M. \& Medzhitov, R. Regulation of phagosome maturation by signals from toll-like receptors. Science 304, 1014-1018 (2004).

7 Roger, T. et al. Protection from lethal gram-negative bacterial sepsis by targeting Tolllike receptor 4. Proc. Natl Acad. Sci. USA 106, 2348-2352 (2009).

8 Savva, A. \& Roger, T. Targeting toll-like receptors: promising therapeutic strategies for the management of sepsis-associated pathology and infectious diseases. Front. Immunol. 4, 387 (2013).

9 Pasquale, T. R. \& Tan, J. S. Nonantimicrobial effects of antibacterial agents. Clin. Infect. Dis. 40, 127-135 (2005).

10 Tauber, S. C. \& Nau, R. Immunomodulatory properties of antibiotics. Curr. Mol. Pharmacol. 1, 68-79 (2008). 
11 Bode, C. et al. Antibiotics regulate the immune response in both presence and absence of lipopolysaccharide through modulation of Toll-like receptors, cytokine production and phagocytosis in vitro. Int. Immunopharmacol. 18, 27-34 (2014).

12 Giamarellos-Bourboulis, E. J. et al. Immunomodulatory clarithromycin treatment of experimental sepsis and acute pyelonephritis caused by multidrug-resistant Pseudomonas aeruginosa. Antimicrob. Agents Chemother. 48, 93-99 (2004).

13 Ivetic Tkalcevic, V. et al. Anti-inflammatory activity of azithromycin attenuates the effects of lipopolysaccharide administration in mice. Eur. J. Pharmacol. 539, 131-138 (2006).

14 Giamarellos-Bourboulis, E. J. et al. Effect of clarithromycin in patients with sepsis and ventilator-associated pneumonia. Clin. Infect. Dis. 46, 1157-1164 (2008).

15 Bassetti, M., Ginocchio, F. \& Giacobbe, D. R. New approaches for empiric therapy in Gram-positive sepsis. Minerva Anestesiol. 77, 821-827 (2011).

16 Aziz, M. et al. Current trends in inflammatory and immunomodulatory mediators in sepsis. J. Leukoc. Biol. 93, 329-342 (2013).

17 Martin, J. H. et al. Therapeutic monitoring of vancomycin in adult patients: a consensus review of the American Society of Health-System Pharmacists, the Infectious Diseases Society of America, and the Society Of Infectious Diseases Pharmacists. Clin. Biochem. Rev. 31, 21-24 (2010).

18 Myrianthefs, P. et al. Serum and cerebrospinal fluid concentrations of linezolid in neurosurgical patients. Antimicrob. Agents Chemother. 50, 3971-3976 (2006).

$19 \mathrm{Kim}, \mathrm{A}$. et al. In vivo microdialysis study of the penetration of daptomycin into soft tissues in diabetic versus healthy volunteers. Antimicrob. Agents Chemother. 52, 3941-3946 (2008).

20 English, B. K., Maryniw, E. M., Talati, A. J. \& Meals, E. A. Diminished macrophage inflammatory response to Staphylococcus aureus isolates exposed to daptomycin versus vancomycin or oxacillin. Antimicrob. Agents Chemother. 50, 2225-2227 (2006).

21 lyori, M. et al. Resveratrol modulates phagocytosis of bacteria through an NF-kappaBdependent gene program. Antimicrob. Agents Chemother. 52, 121-127 (2008).

22 Gruger, T. et al. Negative impact of linezolid on human neutrophil functions in vitro. Chemotherapy 58, 206-211 (2012)

23 Wilcox, M. H. et al. Complicated skin and skin-structure infections and catheter-related bloodstream infections: noninferiority of linezolid in a phase 3 study. Clin. Infect. Dis. 48, 203-212 (2009).

24 Calvano, J. E. et al. Modulation of the lipopolysaccharide receptor complex (CD14 TLR4, MD-2) and toll-like receptor 2 in systemic inflammatory response syndromepositive patients with and without infection: relationship to tolerance. Shock 20 415-419 (2003).
25 Levy, 0. et al. Selective impairment of TLR-mediated innate immunity in human newborns: neonatal blood plasma reduces monocyte TNF-alpha induction by bacterial lipopeptides, lipopolysaccharide, and imiquimod, but preserves the response to R-848. J. Immunol. 173, 4627-4634 (2004)

26 Devaraj, S., Venugopal, S. K., Singh, U. \& Jialal, I. Hyperglycemia induces monocytic release of interleukin- 6 via induction of protein kinase $c$-\{alpha\} and -\{beta\}. Diabetes 54, 85-91 (2005).

27 Ziegeler, S. et al. Antibiotics modulate the stimulated cytokine response to endotoxin in a human ex vivo, in vitro model. Acta Anaesthesiol. Scand. 50, 1103-1110 (2006).

28 Iyer, S. S. et al.Mitochondrial cardiolipin is required for Nlrp3 inflammasome activation. Immunity 39, 311-323 (2013).

29 Garcia-Roca, P. et al. Linezolid diminishes inflammatory cytokine production from human peripheral blood mononuclear cells. Arch. Med. Res. 37, 31-35 (2006).

30 Thallinger, C. et al. Daptomycin does not exert immunomodulatory effects in an experimental endotoxin model of human whole blood. Pharmacology $\mathbf{8 1}$, 57-62 (2008).

31 Bailly, S. et al. Quinolone-induced differential modification of IL-1 alpha and IL-1 beta production by LPS-stimulated human monocytes. Cell. Immunol. 128 277-288 (1990).

32 Bailly, S., Fay, M., Ferrua, B. \& Gougerot-Pocidalo, M. A. Ciprofloxacin treatment in vivo increases the ex vivo capacity of lipopolysaccharide-stimulated human monocytes to produce IL-1, IL-6 and tumour necrosis factor-alpha. Clin. Exp. Immunol. 85, 331-334 (1991).

33 Kelesidis, T. The interplay between daptomycin and the immune system. Front. Immunol. 5, 52 (2014).

(c) (1) (-) $\Theta$ This work is licensed under a Creative Commons Attribution-NonCommercial-NoDerivs $\quad 4.0$ International License. The images or other third party material in this article are included in the article's Creative Commons license, unless indicated otherwise in the credit line; if the material is not included under the Creative Commons license, users will need to obtain permission from the license holder to reproduce the material. To view a copy of this license, visit http://creativecommons.org/licenses/bync-nd/4.0/

Supplementary Information accompanies the paper on The Journal of Antibiotics website (http://www.nature.com/ja) 